\title{
BMJ Open Current status and progression of lower urinary tract symptoms in Chinese male patients: the protocol and rationale for a nationwide, hospital-based, prospective, multicentre study
}

Qi-Xiang Song (D) , ${ }^{1}$ Yi Zhang, ${ }^{2}$ Xiaofei Ye, ${ }^{3}$ Wei Xue, ${ }^{4}$ Chuanliang Xu, ${ }^{1}$ Jing Xu, ${ }^{5}$ Paul Abrams, ${ }^{6}$ Yinghao Sun, ${ }^{1}$ On behalf of the Prostatic Obstruction Investigation Team (POInT)

To cite: Song $Q-X$, Zhang $Y$, Ye $X$, et al. Current status and progression of lower urinary tract symptoms in Chinese male patients: the protocol and rationale for a nationwide, hospital-based, prospective, multicentre study. BMJ Open 2019;9:e028378. doi:10.1136/ bmjopen-2018-028378

- Prepublication history for this paper is available online. To view these files please visit the journal online (http://dx.doi. org/10.1136/bmjopen-2018028378).

Q-XS and YZ contributed equally.

Received 05 December 2018

Revised 16 July 2019

Accepted 18 July 2019
Check for updates

(C) Author(s) (or their employer(s)) 2019. Re-use permitted under CC BY-NC. No commercial re-use. See rights and permissions. Published by BMJ.

For numbered affiliations see end of article.

Correspondence to

Dr Yinghao Sun;

sunyhsmmu@126.com

\section{ABSTRACT}

Introduction It has become increasingly evident that the male lower urinary tract symptoms (LUTS) may not only be caused by abnormalities in one or more components of the lower urinary tract, but also be secondary to a range of systemic disorders. The primary aim of this study is to evaluate the features of urological and systemic disorders among middle-aged and elderly male LUTS patients in China. The second aim is to investigate the changes of both conditions after interventions through a 2-year prospective follow-up.

Methods and analysis This nationwide, hospital-based, multicentre cohort study was designed and initiated by the Prostatic Obstruction Investigation Team which is an international multidisciplinary academic group focusing on the management of male LUTS. An expectation of 11 500 eligible subjects from 23 authorised urological centres across the mainland of China will be recruited with a baseline assessment and data collection. Subsequently, subjects will undergo a follow-up for 24 months having been prescribed with oral medications or after being selected for prostate surgery based on the standard practice of each institution as well as the clinician's own experience. All statistical analysis will be performed using SAS V.9.4 and R package.

Ethics and dissemination This study has received ethical approval from the Ethics Committee Boards of Shanghai Changhai Hospital (CHEC2017-244). The results will be reported at scientific conferences and be published on peer-reviewed journals.

Trial registration NCT03394651.

\section{INTRODUCTION}

Lower urinary tract symptoms (LUTS), encompassing storage, voiding and postmicturition symptoms, are common conditions in men that cause significant quality of life and economic burdens. ${ }^{12}$ One of the largest population-based surveys demonstrated that approximately $62.5 \%$ of the general male population in selected countries reported
Strengths and limitations of this study

- This large-scale, nationwide, multicentre trial consists not only of a cross-sectional investigation of men with lower urinary tract symptoms (LUTS), but also the longitudinal follow-up which includes the variation trends of both symptoms and systemic disorders after treatments.

- As an international multidisciplinary academic group, the Prostatic Obstruction Investigation Team is able to provide full-scale support throughout the design, process and outcome assessment of this trial.

- Unlike the previous demographic surveys conducted via internet, telephone or mail based exclusively on self-reports, the hospital-based design of this study will ensure that all clinical information is recorded by trained physicians in-person which maximise the data quality.

- Instead of simply documenting the history of comorbidities, much attention will be paid to clinical laboratory assessments, such as, kidney function, blood lipid and blood glucose and their associations with LUTS.

- Though with robust sample size, our recruited subjects still may not fully represent the status of the whole country, due to potential financial, educational and geographical disparities.

experiencing one or more LUTS and that its prevalence increased with age. ${ }^{3}$ Recently, an investigation of an Asian population revealed that the overall prevalence of male LUTS was $62.8 \%$, with the storage, voiding and postmicturition symptoms reported in $49.9 \%, 45.3 \%$ and $32.3 \%$ of men, respectively. ${ }^{4}$

Although traditionally, an obstructing enlarged prostate was believed to be the main factor giving rise to male LUTS, recent studies have revealed that the aetiology can 
goes beyond the prostate and even beyond urology ${ }^{56}$ For example, urodynamically proven bladder outlet obstruction only accounts for about $50 \%$ of men presenting with LUTS, while the proportion of the rest might be due to bladder dysfunctions, for example, detrusor overactivity during filling or detrusor underactivity during voiding. ${ }^{7}$ Moreover, increasing evidence has suggested the common pathophysiological determinants for the onset of LUTS and the metabolic syndrome, with a complex of disorder including cardiological abnormalities, impaired glucose handling, central obesity, hypertension, microalbuminuria and dyslipidaemia. ${ }^{89}$

In addition, sustained lower urinary tract dysfunction may jeopardise the upper urinary tract. This was evidenced by the findings that among investigated men with benign prostatic obstruction (BPO) and voiding symptoms, with $11 \%$ experiencing elevated serum creatinine level $(>1.4 \mathrm{mg} / \mathrm{dL})$, possibly associated with their BPO. ${ }^{10}$ If assessed using estimated glomerular filtration rate (eGFR), as many as $18.0 \%-31.9 \%$ of patients with BPO presented with moderate to severe kidney function reduction (defined as eGFR lower than $60 \mathrm{~mL} / \mathrm{min} / 1.73$ $\left.\mathrm{m}^{2}\right) .^{1112}$

Therefore, to promote the individualised, integrated and multidisciplinary management of LUTS, the Prostatic
Obstruction Investigation Team (POInT) was established in Shanghai, China in 2017. In the present study, we aim to elucidate the symptom characteristics of LUTS and comorbidities of middle-aged and elderly men in China. We will also evaluate the progression of both conditions through a 2-year prospective follow-up.

\section{METHODS AND ANALYSIS Study design}

We are proposing a nationwide, hospital-based, multicentre, prospective, observational, cohort study initiated by POInT, including 23eligible hospitals across the mainland of China. All urology departments were carefully selected based on their geographical regions, urology department size, past medical service quality and patient volume. The details of participating hospitals are listed in table 1.

It is planned that a total of 11500 eligible men (an average of at least 500 patients per hospital) presenting with LUTS will be recruited over a 24-month period. In study stage I (baseline assessment), after completion of a written informed consent, the baseline assessments will be carried out and the data will be processed by experienced urologists. In stage II (follow-up assessment), those

Table 1 Study locations and participating hospitals

\begin{tabular}{|c|c|c|}
\hline Partners & City/Province & $\begin{array}{l}\text { Geographical } \\
\text { regions }\end{array}$ \\
\hline Department of Urology, Changhai Hospital & Shanghai & Eastern region \\
\hline Department of Urology, Renji Hospital, Shanghai Jiao Tong University & Shanghai & Eastern region \\
\hline Department of Urology, Changzheng Hospital & Shanghai & Eastern region \\
\hline Department of Urology, Shanghai General Hospital & Shanghai & Eastern region \\
\hline Department of Urology, Zhongshan Hospital, Fudan University & Shanghai & Eastern region \\
\hline Department of Urology, Jiangsu Province Hospital & Jiangsu & Eastern region \\
\hline Department of Urology, Zhongda Hospital & Jiangsu & Eastern region \\
\hline Department of Urology, The Second Affiliated Hospital of Nanjing Medical University & Jiangsu & Eastern region \\
\hline Department of Urology, The First Affiliated Hospital of Suzhou University & Jiangsu & Eastern region \\
\hline Department of Urology, Peking University Third Hospital & Beijing & Northern region \\
\hline Department of Urology, Beijing Hospital & Beijing & Northern region \\
\hline Department of Urology, The Second Hospital of Tianjin Medical University & Tianjin & Northern region \\
\hline Department of Urology, Sun Yat-Sen Memorial Hospital & Guangzhou & Southern region \\
\hline Department of Urology, The Third Affiliated Hospital of Sun Yat-Sen University & Guangzhou & Southern region \\
\hline Department of Urology, Zhujiang Hospital & Guangzhou & Southern region \\
\hline Department of Urology, The First Affiliated Hospital of Fujian Medical University & Fuzhou & Southern region \\
\hline Department of Urology, Xinqiao Hospital & Chongqing & Western region \\
\hline Department of Urology, Southwest Hospital & Chongqing & Western region \\
\hline
\end{tabular}



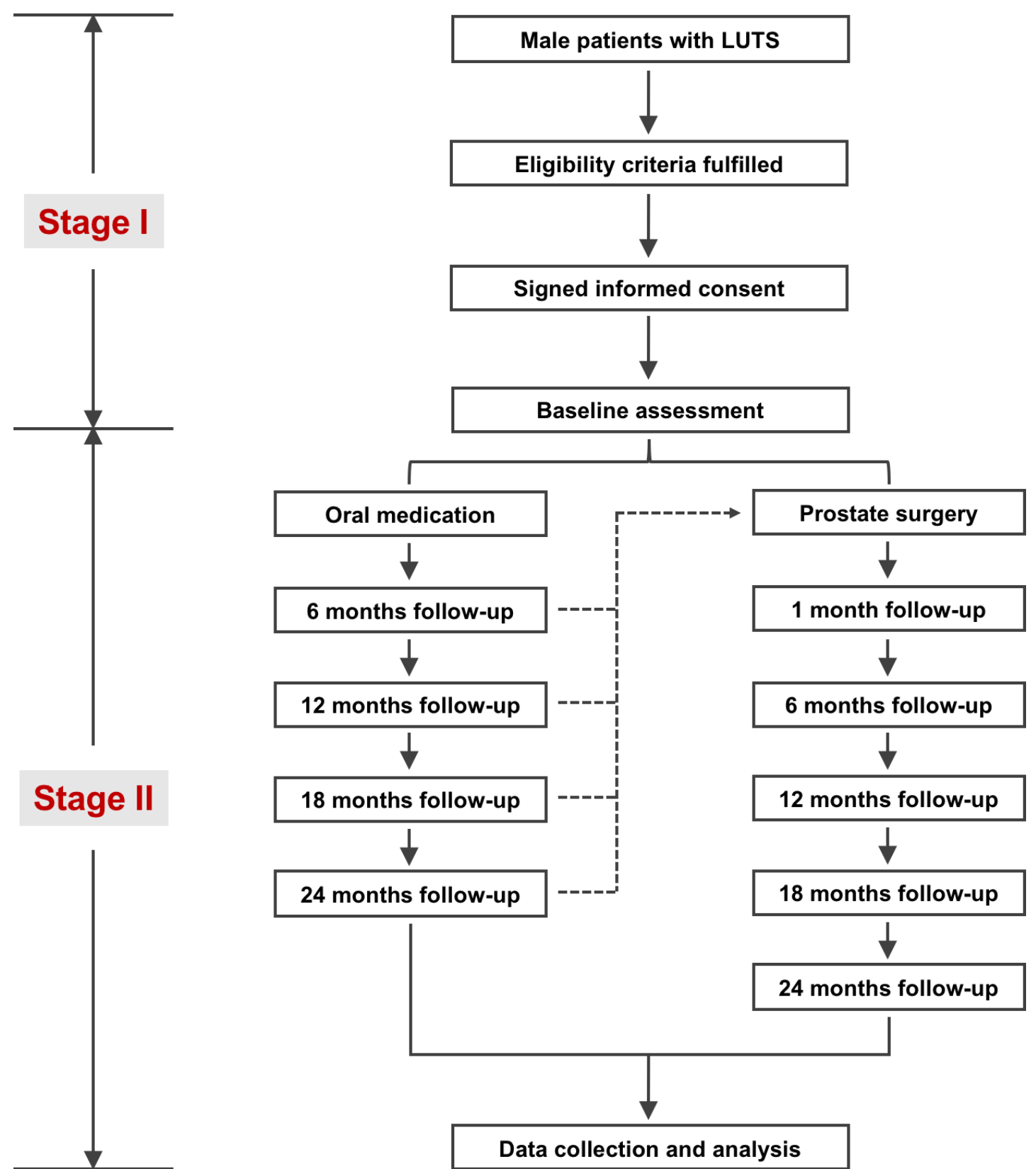

Figure 1 The study flow diagram. During stage I, after checked for eligibility, subjects will be required to sign the informed consent followed by baseline assessment. In the follow-up stage, subjects will receive either oral medications or prostate surgery and will be followed up for 24 months. LUTS, lower urinary tract symptoms.

recruited patients will be prescribed either oral medications or will be selected as candidates for prostate surgery, according to their symptom severity, the presence of complications and the patients' preference. Subjects receiving medical therapy will be followed up every 6 months for 2 years, while those who receiving surgical treatment will be assessed 1 and 6 months post-surgery and then every 6 months for 2 years. Figure 1 gives the study flow diagram that the patients will follow during the course of the study.

\section{Biases/blinding}

Since this is a non-randomised trial and no sham treatment group will be involved, the patients and caregivers will not be blinded to treatments. To minimise the potential biases, the data assessors will be blinded to the initial clinical status and treatment approaches of each subject.

\section{Inclusion and exclusion criteria}

Men at least 45 years of age with LUTS who seek clinical management, in the authorised participating hospitals, will be recruited for further assessments. During the screening visit, the patients' history and basic information will be carefully documented, including chief complaints, medical and surgical history, smoking status, chronic coexisting conditions and current medication usage. Digital rectal examination and serum prostate specific antigen (PSA) will be used to rule out the coexistence of 


\section{Box 1 Inclusion and exclusion criteria}

Inclusion criteria
Male.
Age $\geq 45$ years.
Presenting with lower urinary tract symptoms (LUTS).
Willing to participate in the study and who have signed informed
consent.
Exclusion criteria
LUTS as a result of urethral stricture, stone diseases, chronic pros-
tatitis, active urinary tract infection, space-occupying lesions and
so on.
Diagnosis or suspicion of renal, ureteral, bladder, prostate, urethral
or pelvic tumour.
Known neurogenic or congenital lower urinary tract dysfunction.
Known lower urinary tract or pelvic surgical history.
Existence of anatomical abnormalities of the urinary tract (eg, diver-
ticulum of the bladder or urethra, ectopic ureteral orifice, etc).
Any serious medical condition likely to impede successful com-
pletion of the study (eg, heart failure, myocardial infarction, recent
cerebrovascular accident, etc).
Patients with poor compliance or cognitive competence who are un-
able to follow the study protocol.

prostate cancer. Urinalysis will be performed to exclude haematuria and acute urinary tract infection. Ultrasonography will be used to detect potential urinary tract (both upper and lower urinary tracts) and pelvic abnormalities. Box 1 shows a detailed list of the major inclusion and exclusion criteria.

\section{Interventions}

For subjects allocated to the medicine treatment arm, $\alpha_{1}$-adrenoceptor antagonists and/or $5 \alpha$-reductase inhibitors will be prescribed if the man has predominantly voiding symptoms. Muscarinic receptor antagonists can be added when subjects present with bothersome storage symptoms, with a post-void residual (PVR) volume of less than $150 \mathrm{~mL} .^{13}$ The use of other potential therapies, including beta-3 agonist, phosphodiesterase five inhibitors and Chinese herb medicines, will be carefully documented. All medications will be prescribed in accordance with standard clinical care and will not be altered for this observational study.

Surgeries will be reserved for those with absolute indications for surgery, or who are non-responders to medical treatment, or those who request surgical treatment at baseline. The selection of surgical approach is based on the current guidelines as well as the surgeon's own experience. ${ }^{14}$ Generally, transurethral resection of the prostate is the main approach for subjects whose prostate volume is less than $80 \mathrm{~mL}$. The laser techniques, including holmium laser enucleation, green light vaporisation and thulium laser enucleation/vaporisation, and other minimally invasive methods, such as transurethral microwave therapy, transurethral needle ablation and prostatic urethral lift, will also be included if used.
Procedures will be performed by experienced surgeons using standard techniques.

\section{Primary outcomes}

The primary outcomes will be the changes in International Prostate Symptom Score (IPSS) and maximum flow rate $\left(Q_{\max }\right)$ at 2 years compared with baseline. The IPSS will be assessed using a standard scoring system which has been previously validated by several studies. ${ }^{15} 16$ The $\mathrm{Q}_{\max }$ will be measured during uroflowmetry with a voided volume at least $125 \mathrm{~mL} \cdot{ }^{17}$ Both outcomes will be collected at baseline and 6, 12, 18, 24 months if oral medication is provided, or at 1, 6, 12, 18, 24 months after surgery (table 2).

\section{Secondary outcomes}

The changes of nocturia and sexual function from baseline will be evaluated using standard questionnaires, that is, international consultation on incontinence questionnaire-nocturia quality of life questionnaire and international index of erectile function. ${ }^{18}{ }^{19}$ The subjects will be requested to complete the questionnaires at baseline assessment and then 6, 12, 18, 24 months if oral medication is prescribed, or at 1, 6, 12, 18, 24 months after prostate surgery. To ensure each patient can fully comprehend the items listed on the questionnaires, experts' explanation is available throughout the assessment.

The measurement of prostatic volume will be carried out via transrectal prostate ultrasound with the patient in a left decubitus position and the prostate ellipsoid formula (length $\times$ width $\times$ height $\times \Phi / 6$ ) will be adopted for calculation. ${ }^{20}$ In case of a well-described median lobe, intravesical prostatic protrusion (IPP) will be measured as the distance between the tip of the prostate median lobe and bladder neck. ${ }^{21}$ The prostatic volume and IPP will be measured at baseline assessment and then yearly after oral medication treatment.

The pressure-flow study (PFS) will be conducted in accordance with the International Continence Society Good Urodynamic Practice Guidelines, utilising water filled urethral and rectal catheters for simultaneous measurements of vesical and abdominal pressures. ${ }^{22}$ For patients who require surgical intervention, PFS is used to record $Q_{\max }$ and detrusor pressure at $\mathrm{Q}_{\max }\left(\mathrm{P}_{\text {det }} \mathrm{Q}_{\max }\right)$. Bladder outlet obstruction index and bladder contractility index will be calculated as $P_{\text {det }} Q_{\max }-2 Q_{\max }$ and $P_{\text {det }} Q_{\max }+5$ $Q_{\text {max }}$, respectively. ${ }^{2324}$ The PFS will be performed at baseline or at any time point during follow-up in the medicine treatment arm if surgical intervention is required and will be repeated yearly after surgery.

A variety of serum and urine parameters to document kidney function, and blood lipids and glucose, will be measured with standard methods and commercially available reagents. The eGFR is calculated using both Chronic Kidney Disease Epidemiology Collaboration equation and Modification of Diet in Renal Disease equation for Chinese patients with chronic kidney diseases. ${ }^{25}{ }^{26}$ These data will be measured at baseline and yearly in both arms. 
Table 2 Study timeline

\begin{tabular}{|c|c|c|c|c|c|c|c|c|c|}
\hline \multirow[b]{2}{*}{ Month number } & \multirow{2}{*}{$\begin{array}{l}\text { Baseline } \\
0\end{array}$} & \multicolumn{3}{|c|}{ Follow-up (oral medication arm) } & \multicolumn{5}{|c|}{ Follow-up (prostate surgery arm) } \\
\hline & & 12 & 18 & 24 & 1 & 6 & 12 & 18 & 24 \\
\hline Singed informed consent & $\sqrt{ }$ & & & & & & & & \\
\hline \multicolumn{10}{|l|}{ Basic information } \\
\hline Name & $\sqrt{ }$ & & & & & & & & \\
\hline Age & $\sqrt{ }$ & & & & & & & & \\
\hline BMI & $\sqrt{ }$ & & & & & & & & \\
\hline Education background & $\sqrt{ }$ & & & & & & & & \\
\hline Smoking status & $\sqrt{ }$ & & & & & & & & \\
\hline \multicolumn{10}{|l|}{ History } \\
\hline Chief complains & $\sqrt{ }$ & & & & & & & & \\
\hline Medical history & $\sqrt{ }$ & & & & & & & & \\
\hline Current medication status & $\sqrt{ }$ & & & & & & & & \\
\hline
\end{tabular}

Questionnaires

\begin{tabular}{|c|c|c|c|c|c|c|c|c|c|c|}
\hline IPSS & $\sqrt{ }$ & $\sqrt{ }$ & $\sqrt{ }$ & $\sqrt{ }$ & $\sqrt{ }$ & $\sqrt{ }$ & $\sqrt{ }$ & $\sqrt{ }$ & $\sqrt{ }$ & $\sqrt{ }$ \\
\hline ICIQ-N-QoL & $\sqrt{ }$ & $\sqrt{ }$ & $\sqrt{ }$ & $\sqrt{ }$ & $\sqrt{ }$ & $\sqrt{ }$ & $\sqrt{ }$ & $\sqrt{ }$ & $\sqrt{ }$ & $\sqrt{ }$ \\
\hline IIEF & $\sqrt{ }$ & $\sqrt{ }$ & $\sqrt{ }$ & $\sqrt{ }$ & $\sqrt{ }$ & $\sqrt{ }$ & $\sqrt{ }$ & $\sqrt{ }$ & $\sqrt{ }$ & $\sqrt{ }$ \\
\hline Transrectal ultrasound & $\sqrt{ }$ & & $\sqrt{ }$ & & $\sqrt{ }$ & & & & & \\
\hline $\begin{array}{l}\text { Uroflowmetry and post void } \\
\text { residual }\end{array}$ & $\sqrt{ }$ & $\sqrt{ }$ & $\sqrt{ }$ & $\sqrt{ }$ & $\sqrt{ }$ & $\sqrt{ }$ & $\sqrt{ }$ & $\sqrt{ }$ & $\sqrt{ }$ & $\sqrt{ }$ \\
\hline Pressure-flow study & $\Delta$ & & & & & & & $\Delta$ & & $\Delta$ \\
\hline Urinalysis & $\sqrt{ }$ & $\sqrt{ }$ & $\sqrt{ }$ & $\sqrt{ }$ & $\sqrt{ }$ & $\sqrt{ }$ & $\sqrt{ }$ & $\sqrt{ }$ & $\sqrt{ }$ & $\sqrt{ }$ \\
\hline Serum PSA & $\sqrt{ }$ & & $\sqrt{ }$ & & $\sqrt{ }$ & & & $\sqrt{ }$ & & $\sqrt{ }$ \\
\hline Blood pressure & $\sqrt{ }$ & $\sqrt{ }$ & $\sqrt{ }$ & $\sqrt{ }$ & $\sqrt{ }$ & $\sqrt{ }$ & $\sqrt{ }$ & $\sqrt{ }$ & $\sqrt{ }$ & $\sqrt{ }$ \\
\hline
\end{tabular}

Renal function

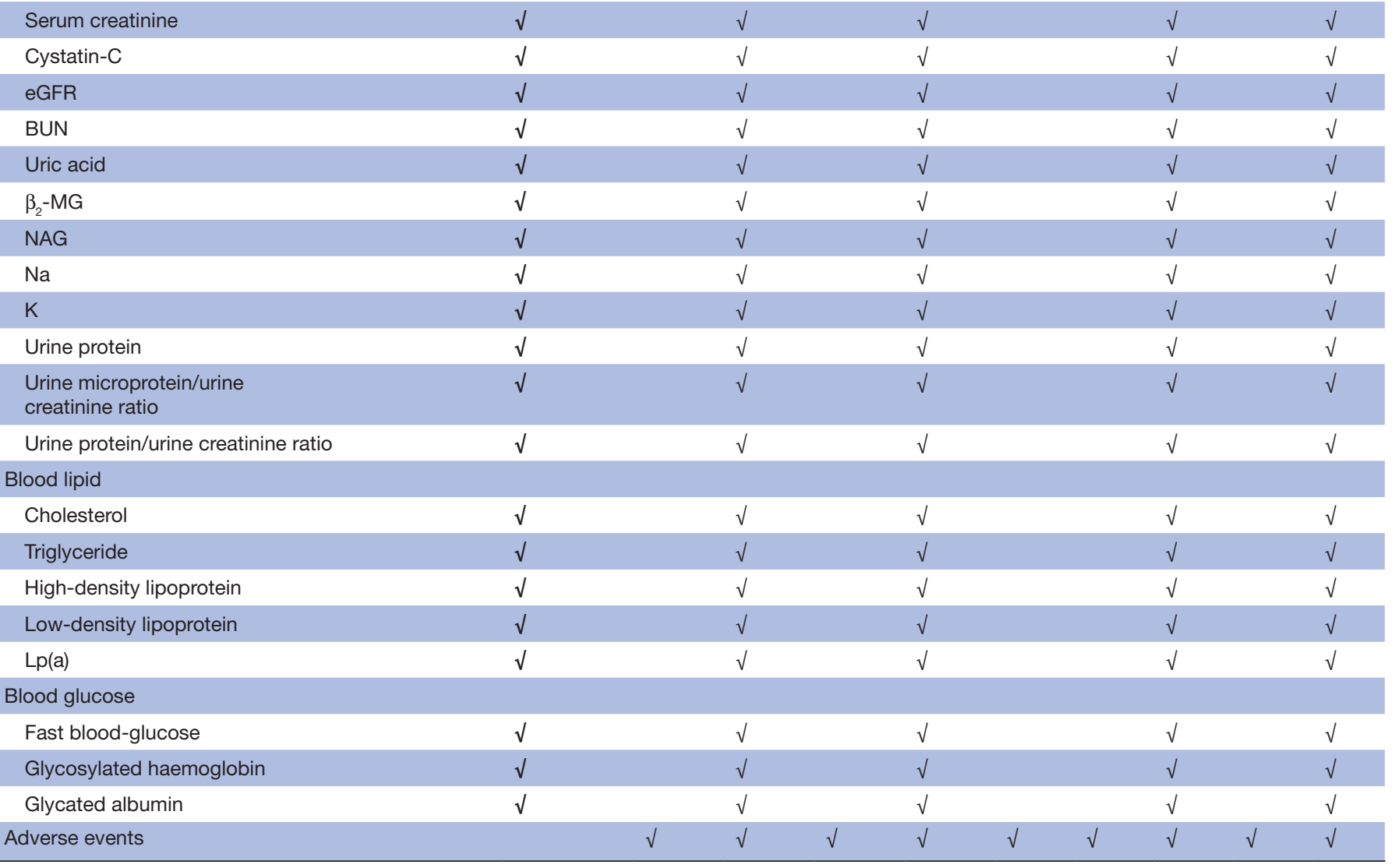


Table 2 Continued

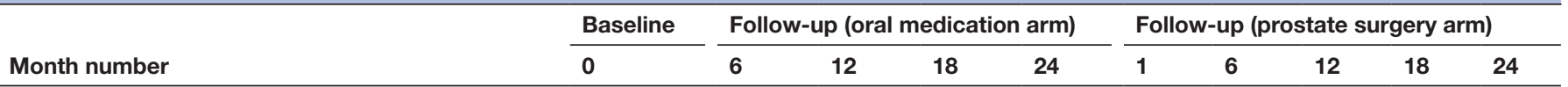

BMI, body mass index; BUN, blood urea nitrogen; eGFR, estimated glomerular filtration rate; ICIQ-N-QoL, international consultation on incontinence questionnairenocturia quality of life; IIEF, international index of erectile function; IPSS, International Prostate Symptom Score; NAG, N-acetyl-beta-D-glucosaminidase; PSA, prostate specific antigen.

The blood pressure will be measured by either mercury or electronic sphygmomanometer when the subjects are relaxed. Hypertension is defined as the systolic/diastolic blood pressures of 140/90 $\mathrm{mm} \mathrm{Hg}$ or more, any use of antihypertensive medication in the past 2 weeks, or any self-reported history of hypertension. The blood pressure will be measured at baseline and 6, 12, 18, 24 months if oral medication is provided or 1, 6, 12, 18, 24 months after surgery.

Other medical comorbidities (such as diabetes mellitus, chronic obstructive pulmonary disease, coronary heart disease, Parkinson's disease, etc) will be recorded based on patients' self-report or their past medical records during the initial recruitment. PVR volume will be assessed by ultrasound at baseline and each time point during follow-up. PSA will be measured at baseline and then yearly in both treatment arms. Adverse effects relating to the medicine or surgery will be documented at each time point during follow-up.

\section{Sample size calculation}

The formula for sample size estimation was as follows: where,

n: estimated sample size;

z: liked to $95 \%$ CI, $\alpha=0.05, \mathrm{z}=1.96$;

$\sigma: \mathrm{SD}$

$\delta$ : relative desired precision.

Based on previous experience, the SD of IPSS and $Q_{\max }$ was 8.1 and $9.5 \mathrm{~m} / \mathrm{s}$, respectively. ${ }^{27}$ The relative desired precision for IPSS and $Q_{\max }$ in this study was one for both indices. The minimal sample size was estimated to be 253 and 345 , for IPSS and $Q_{\max }$, respectively. In order to get the precise estimation of both indices, the minimal sample size was set to 345 . With an expanded sample size of $120 \%$, the minimal sample size was adjusted to be 414 . In fact, a total of 11500 eligible men are expected to be recruited in this study. This will mean that analysis of the primary outcomes can be studied in relation to other factors such as comorbidities.

\section{Statistical methods}

All statistical analysis will be performed using SAS V.9.4 and $\mathrm{R}$ package. Statistical significance will be defined as $p<0.05$. The demographic information, laboratory test data, comorbidity status and so on will be analysed. Continuous variables were presented as mean $\pm \mathrm{SD}$ compared using the independent sample t-test assuming a normal distribution, whereas categorical variables were expressed as frequency compared using the Pearson $\chi^{2}$ test. Pearson linear correlation will be calculated between two continuous variables, such as, age, body mass index, PSA and LUTS related parameters. Multivariate linear regression will be performed to determine the independent predictors of LUTS characteristics and severity. A stepwise selection technique in binary logistic regression model will be used for multivariate analysis to select factors associated with binary variables (such as the two treatment selections: oral medication and prostate surgery, and comorbidity status). The differences in clinical profiles, therapeutic approaches and outcomes between two cohorts (oral medication and prostate surgery) will be analysed after propensity score matching. In addition, subgroup analysis will be performed, such as different IPSS value groups.

\section{Quality control}

The site investigators and staff members must complete a training programme regarding the design and process of the study for standardisation and data consistency. All participants have agreed to vouch for the accuracy of the data and adherence of the sites to the study protocol. For quality control of the serum and urine sample tests, clinical laboratories in each site must meet the ISO15189 and/or CAP requirements. An independent data and safety supervising board will monitor the trial for preciseness and scientific integrity. Interim meetings will be held every 3-6 months to ensure the protocol is being followed and data is collected properly.

\section{Patients and public involvement statement}

The development of current research protocol and outcome measures was informed by the symptoms and characteristics of male LUTS patients, with the notion that LUTS is not always related to the prostate, but rather should be viewed systemically. Although patients did not participate during the phase of study design, their concerns and questions were addressed during recruitment and study implementation.

\section{ETHICS AND DISSEMINATION}

This study has received ethical approval from the Ethics Committee Boards of Shanghai Changhai Hospital (CHEC2017-244). The principles of the Declaration of Helsinki and Good Clinical Practice will be complied with. The study will be subjected to the Strengthening the Reporting of Observational Studies in Epidemiology reporting guidelines. ${ }^{28}$ Written informed consent will be obtained from all eligible subjects in the trial. All personal information and clinical results will be collected and 
preserved via an encrypted internet database which can only be accessed via authorised personnel. The system will be maintained at the network centre of Naval Medical University and data will be backed up into the hard drive periodically. The results will be reported at scientific conferences and published on peer-reviewed journals.

\section{DISCUSSION}

LUTS is a variable multisymptom condition with various potential contributing aetiological causes and significant symptom overlap. ${ }^{5}$ Therefore, the importance of implementing a more holistic perspective to the interpretation of LUTS is likely to allows us to diagnose and manage our patients more effectively. The primary goal of the present study is to investigate the features of LUTS, and its associated systemic comorbid conditions among Chinese men. We will also assess the progression of symptoms and comorbidities, after both oral medication and surgical intervention during a 24-month follow-up period.

Several strengths should be pointed out in the design of this study. First, POInT is an international multidisciplinary academic group with cooperative efforts between urology, nephrology, medical health and biostatistics departments. The teamwork provides full-scale support throughout the design, process and outcome assessment of this trial. Second, unlike the previous demographic surveys conducted via internet, telephone or mail based exclusively on self-reports, we propose a hospital-based study with a large sample size and wide coverage. ${ }^{4930}$ All patients' data, including subjective and objective outcomes, will be evaluated and recorded by trained physicians in-person which maximally ensures the quality of data. Third, with the participation of the hospitals from multiple geographic regions of China, this study makes it likely that prognostic outcomes will be representative of all China's population reflecting local medical practice. Fourth, this is a two-step trial providing insight into not only the cross-sectional investigation of men with LUTS at baseline, but also the longitudinal variations and trends of symptoms and systemic disorders following medication or invasive treatment. The outcomes could shed light on the potential associations between the changes of LUTS and even the development of future comorbidities following a specific treatment. In addition, instead of simply documenting the history of comorbidities, much attention will be paid to clinical laboratory assessments, especially, kidney function, blood lipids and blood glucose, and their associations with LUTS. Such information might be used as predictive factors for clinicians to carry out systemic evaluation and treatment, in the future.

There are some limitations to consider in our design. Though the sample size is large, our cohort may not fully represent Chinese middle-aged and elderly male population with LUTS. This could due partially to our recruitment being hospital-based which may be biassed towards those who suffer from moderated to severe LUTS, since patients may not present to the clinics unless the symptoms are bothersome. Therefore, unlike population-based or community-based surveys, patients with merely mild symptoms may not be included. However, we believe that men with mild, non-bothersome symptoms are unlikely to present to their doctor. Further potential disadvantages are that individuals who have low educational level or live in remote rural areas with limited access to the selected hospitals, may not be included. However, such patients might have difficulties in fully comprehending and completing the symptom questionnaires and may have poor compliance during follow-up. Blood and urine samples are measured in each authorised hospital rather than in one central laboratory. Therefore, the possibility of bias and variation between laboratories may exist, although this is unlikely as every clinical laboratory, in each city, meets the ISO15189 and/or CAP requirements.

This large-scale nationwide cohort study is designed to investigate concurrently the characteristics of Chinese men with LUTS as well as changes in urological symptoms and systemic comorbid conditions following either medication or surgical intervention. The results of this study will shed light on the global clinical management of each patient, provide evidence for basic science research on the causative mechanisms of LUTS, and may change the way Chinese men with LUTS are treated in future.

\section{TRIAL STATUS}

Four consensus meetings were held and the investigators in each site have completed the protocol training programme. The study has been open for recruitment since April 2018 and is currently ongoing.

\section{Author affiliations}

${ }^{1}$ Department of Urology, Changhai Hospital, Shanghai, China

${ }^{2}$ Department of Military Health Service, Naval Medical University, Shanghai, China

${ }^{3}$ Department of Health Statistics, Naval Medical University, Shanghai, China

${ }^{4}$ Department of Urology, Renji Hospital, Shanghai Jiao Tong University, Shanghai,

China

${ }^{5}$ Department of Nephrology, Changhai Hospital, Shanghai, China

${ }^{6}$ Bristol Urological Institute, Southmead Hospital, Bristol, UK

Acknowledgements We are grateful to the participating institutes of POInT and all the patient advisers who are collaborating in this study.

Contributors $Q-X S, P A$ and $Y S$ designed the study. WX, CX and JX revised the protocol and held the consensus meetings. $Q-X S, Y Z$ and $X Y$ are responsible for the establishment of database as well as statistical design. Q-XS and YZ drafted the manuscript. WX, CX, PA and YS critically revised the manuscript. All authors have approved the final version of the manuscript.

Funding This work was supported by National Natural Science Foundation of China (N0. 81500579) and Translational Medicine Research Project of Naval Medical University (N0. 2017JZ44).

Competing interests PA is a consultant for Pfizer, Astellas, Ferring, Ipsen and a lecturer for Astellas, Pfizer, Ferring, Sanofi, Sun Pharma, Coloplast, Pierre Fabre. Other authors have no real or potential conflicts of interest with respect to the trial, authorship and/or publication of this article.

Patient consent for publication Parental/guardian consent obtained Provenance and peer review Not commissioned; externally peer reviewed. Open access This is an open access article distributed in accordance with the Creative Commons Attribution Non Commercial (CC BY-NC 4.0) license, which 
permits others to distribute, remix, adapt, build upon this work non-commercially, and license their derivative works on different terms, provided the original work is properly cited, appropriate credit is given, any changes made indicated, and the use is non-commercial. See: http://creativecommons.org/licenses/by-nc/4.0/.

ORCID iD

Qi-Xiang Song http://orcid.org/0000-0001-6393-9275

\section{REFERENCES}

1. Coyne KS, Sexton CC, Irwin DE, et al. The impact of overactive bladder, incontinence and other lower urinary tract symptoms on quality of life, work productivity, sexuality and emotional wellbeing in men and women: results from the EPIC study. BJU Int 2008;101:1388-95.

2. Coyne KS, Wein AJ, Tubaro A, et al. The burden of lower urinary tract symptoms: evaluating the effect of LUTS on health-related quality of life, anxiety and depression: EpiLUTS. BJU Int 2009;103(Suppl 3):4-11.

3. Irwin DE, Milsom I, Kopp Z, et al. Prevalence, severity, and symptom bother of lower urinary tract symptoms among men in the EPIC study: impact of overactive bladder. Eur Urol 2009;56:14-20.

4. Chapple C, Castro-Diaz D, Chuang Y-C, et al. Prevalence of lower urinary tract symptoms in China, Taiwan, and South Korea: results from a cross-sectional, population-based study. Adv Ther 2017;34:1953-65.

5. Chapple CR, Wein AJ, Abrams P, et al. Lower urinary tract symptoms revisited: a broader clinical perspective. Eur Urol 2008;54:563-9.

6. Song Q, Abrams P, Sun Y. Beyond prostate, beyond surgery and beyond urology: The '3Bs' of managing non-neurogenic male lower urinary tract symptoms. Asian J Urol 2019;6:169-73.

7. Soler R, Andersson K-E, Chancellor MB, et al. Future direction in pharmacotherapy for non-neurogenic male lower urinary tract symptoms. Eur Urol 2013;64:610-21.

8. Denys M-A, Anding R, Tubaro A, et al. Lower urinary tract symptoms and metabolic disorders: ICI-RS 2014. Neurourol Urodyn 2016;35:278-82.

9. Vignozzi L, Gacci M, Maggi M. Lower urinary tract symptoms, benign prostatic hyperplasia and metabolic syndrome. Nat Rev Urol 2016;13:108-19.

10. Gerber GS, Goldfischer ER, Karrison TG, et al. Serum creatinine measurements in men with lower urinary tract symptoms secondary to benign prostatic hyperplasia. Urology 1997;49:697-702.

11. Hong SK, Lee ST, Jeong SJ, et al. Chronic kidney disease among men with lower urinary tract symptoms due to benign prostatic hyperplasia. BJU Int 2010;105:1424-8.

12. Yamasaki T, Naganuma T, Iguchi T, et al. Association between chronic kidney disease and small residual urine volumes in patients with benign prostatic hyperplasia. Nephrology 2011;16:335-9.

13. Abrams P, Kaplan S, De Koning Gans HJ, et al. Safety and tolerability of tolterodine for the treatment of overactive bladder in men with bladder outlet obstruction. J Urol 2006;175:999-1004.

14. Gravas S, Cornu JN, Drake MJ. EAU guidelines on management of non-neurogenic male lower urinary tract symptoms (LUTS) incl.
Benign Prostatic Obstruction. European Association of Urology, 2018.

15. Choi EPH, Lam CLK, Chin W-Y. Validation of the International prostate symptom score in Chinese males and females with lower urinary tract symptoms. Health Qual Life Outcomes 2014;12:1.

16. Wong $\mathrm{CK}-\mathrm{H}$, Choi EP-H, Chan SW-H, et al. Use of the International prostate symptom score (IPSS) in Chinese male patients with benign prostatic hyperplasia. Aging Male 2017;20:241-9.

17. Barry MJ, Fowler FJ, O'Leary MP, et al. The American urological association symptom index for benign prostatic hyperplasia. The measurement Committee of the American urological association. $J$ Urol 1992;148:1549-57.

18. Abraham L, Hareendran A, Mills IW, et al. Development and validation of a quality-of-life measure for men with nocturia. Urology 2004;63:481-6.

19. Rosen RC, Riley A, Wagner G, et al. The International index of erectile function (IIEF): a multidimensional scale for assessment of erectile dysfunction. Urology 1997;49:822-30.

20. Loch AC, Bannowsky A, Baeurle L, et al. Technical and anatomical essentials for transrectal ultrasound of the prostate. World J Urol 2007;25:361-6.

21. Mehraban D. Clinical value of intravesical prostatic protrusion in the evaluation and management of prostatic and other lower urinary tract diseases. Asian J Urol 2017:4:174-80.

22. Schäfer W, Abrams P, Liao L, et al. Good urodynamic practices: uroflowmetry, filling cystometry, and pressure-flow studies. Neurourol Urodyn 2002;21:261-74.

23. Lewis P, Abrams P. Urodynamic protocol and central review of data for clinical trials in lower urinary tract dysfunction. BJU Int 2000;85(Suppl 1):20-30.

24. Abrams P. Bladder outlet obstruction index, bladder contractility index and bladder voiding efficiency: three simple indices to define bladder voiding function. BJU Int 1999;84:14-15.

25. Inker LA, Eckfeldt J, Levey AS, et al. Expressing the CKD-EPI (chronic kidney disease epidemiology collaboration) cystatin C equations for estimating GFR with standardized serum cystatin C values. Am J Kidney Dis 2011;58:682-4.

26. Ma Y-C, Zuo L, Chen J-H, et al. Modified glomerular filtration rate estimating equation for Chinese patients with chronic kidney disease. J Am Soc Nephrol 2006;17:2937-44.

27. Kang M, Kim M, Choo MS, et al. Urodynamic features and significant predictors of bladder outlet obstruction in patients with lower urinary tract Symptoms/Benign prostatic hyperplasia and small prostate volume. Urology 2016;89:96-102.

28. von Elm E, Altman DG, Egger M, et al. The strengthening the reporting of observational studies in epidemiology (STROBE) statement: guidelines for reporting observational studies. The Lancet 2007;370:1453-7.

29. Irwin DE, Milsom I, Hunskaar S, et al. Population-Based survey of urinary incontinence, overactive bladder, and other lower urinary tract symptoms in five countries: results of the EPIC study. Eur Urol 2006;50:1306-15. discussion 1314-5.

30. Seim A, Hoyo C, Ostbye T, et al. The prevalence and correlates of urinary tract symptoms in Norwegian men: the HUNT study. BJU Int 2005;96:88-92. 